\title{
Point-of-Care HIV Testing and Linkage in an Urban Cohort in the Southern US
}

\author{
Anne Zinski, ${ }^{1}$ Sarah M. Dougherty, ${ }^{1}$ Ashutosh Tamhane, ${ }^{2}$ \\ Kelly L. Ross-Davis, ${ }^{3}$ and James L. Raper ${ }^{4}$ \\ ${ }^{1}$ University of Alabama at Birmingham School of Medicine, Division of Infectious Diseases, 845 19th Street South, \\ Bevill Biomedical Research Building 206B, Birmingham, AL 35294-2170, USA \\ ${ }^{2}$ University of Alabama at Birmingham, School of Public Health, Department of Biostatistics, 1665 University Boulevard, \\ RPHB 514-A, Birmingham, AL 35294, USA \\ ${ }^{3}$ University of Alabama at Birmingham, School of Medicine, Division of Infectious Diseases, 908 20th Street South, \\ CCB 188, Birmingham, AL 35294-2050, USA \\ ${ }^{4}$ University of Alabama at Birmingham, School of Medicine, Division of Infectious Diseases, \\ 908 20th Street South, CCB 245, Birmingham, AL 35294-2050, USA
}

Correspondence should be addressed to Anne Zinski; azinski@uab.edu

Received 15 March 2013; Revised 14 June 2013; Accepted 23 July 2013

Academic Editor: Bryce D. Smith

Copyright (C) 2013 Anne Zinski et al. This is an open access article distributed under the Creative Commons Attribution License, which permits unrestricted use, distribution, and reproduction in any medium, provided the original work is properly cited.

\begin{abstract}
The Southern states experience the highest rates of HIV and AIDS in the US, and point-of-care (POC) testing outside of primary care may contribute to status awareness in medically underserved populations in this region. To evaluate POC screening and linkage to care at an urban south site, analyses were performed on a dataset of 3,651 individuals from an integrated rapid-result HIV testing and linkage program to describe this test-seeking cohort and determine trends associated with screening, results, and linkage to care. Four percent of the population had positive results. We observed significant differences by test result for age, race and gender, reported risk behaviors, test location, and motivation for screening. The overall linkage rate was $86 \%$, and we found significant differences for clients who were linked to HIV care versus persons whose linkage could not be confirmed with respect to race and gender, location, and motivation. The linkage rate for POC testing that included a comprehensive intake visit and colocated primary care services for in-state residents was $97 \%$. Additional research on integrated POC screening and linkage methodologies that provide intake services at time of testing is essential for increasing status awareness and improving linkage to HIV care in the US.
\end{abstract}

\section{Introduction}

Prevention and treatment of Human Immunodeficiency Virus (HIV) infection have evolved through important advances in the field, and the number of new HIV infections in the United States has steadied near 50,000 cases annually $[1,2]$. However one-fifth of adults and up to one half of HIV-infected young persons in the United States (US) are not aware of their positive status [3-9]. Considering these statistics, the Centers for Disease Control and Prevention (CDC) released revised recommendations for routine HIV testing during medical visits in US primary care settings [3]; but hurdles to healthcare-based HIV testing still exist at policy, organizational, and individual levels in the Unites States and abroad [10-15]. The recommendations for widespread implementation of standardized opt-out testing in US primary care settings were revised in 2011. Draft revisions in 2013 $[16,17]$ may address challenges associated with inadequate healthcare, targeted screening, and inaccuracies of individual risk perception [18-20]. As approximately $20 \%$ of adults with at least one prior HIV test report a recent (within last 12 months) test in venues outside of primary care offices [14], determining the factors associated with point-of-care (POC) test seeking is an important tactic for expanding serostatus awareness, particularly for medically underserved persons and those who do not have regular contact with the healthcare system. For these Americans, access to rapid, 
noninvasive HIV screening with expeditious result delivery and immediate linkage to care outside of opt-out medical settings is a distinct and invaluable opportunity to enter the HIV care continuum.

Following the 2001 issuance of The Serostatus Approach to Fighting the Epidemic (SAFE) initiative, which highlighted individual HIV status awareness as a catalyst for reducing transmission [21], innovative HIV screening strategies that emphasized broader availability of tests and test results became a public health priority. In 2004, the US Food and Drug Administration (FDA) approved the first POC HIV 1/2 antibody test, which received a Clinical Laboratory Improvement Amendments (CLIA) waiver for oral fluid specimen use outside of phlebotomy laboratories [22, 23]. Though limited in detection of acutely acquired HIV infection, noninvasive POC methodology established increased screening acceptance, particularly among young and lower risk persons [10, 24]. Rapid testing technology also allowed for merged testing and linkage to care services, as trained HIV test counselors could deliver results with individualized linkage, along with pre- and posttest counseling, during one comprehensive visit. This technological advance eliminated multisession presentation to a medical facility with return for separate results, posttest counseling, and referral and linkage visits, which would streamline HIV care services for transient and hard to reach persons.

Thorough and swift pre- and posttest counseling at POC, including linkage or clinic liaison services for persons who test HIV positive, may be a crucial component in the National HIV/AIDS Strategy (NHAS) plan to increase serostatus awareness to $90 \%$ by 2015 [25]. Evidence supports that persons aware of their HIV infection are less likely to transmit to uninfected persons, and that learning one's positive status may prompt initiation of risk reduction behaviors, including disclosure to primary partners [21, 26-29]. Additionally, as serostatus awareness is the first step in scientifically proven HIV treatment and prevention strategies, using either preexposure prophylaxis (PrEP), treatment as prevention, or antiretroviral therapy to improve clinical outcomes, POC serostatus awareness campaigns that aim to link persons to timely treatment play a pivotal role for the test-and-treat initiative at the commencement of the HIV treatment cascade $[30,31]$.

The National HIV/AIDS Strategy goal of improving timely linkage to care for newly diagnosed individuals from $65 \%$ to $85 \%$ motivated researchers and healthcare professionals to develop enhanced tactics to increase early linkage [25]. Recent literature asserts that achieving these goals can increase life expectancy and may prove to be cost effective [32]. In addition to recommendations for surveillance monitoring, initiation of early treatment, and treatment as prevention tactics, POC screening programs that emphasize results counseling and appropriate linkage strategies, while addressing factors that delay linkage to care, may improve HIV outcomes [33, 34].

In this study, we document successes and challenges of an integrated POC screening and linkage program in an urban setting in the Southern Unites States. In addition, we describe the HIV test-seeking cohort (years 2007-2012) according to test result and yearly trends. Further, we examine how clients who test positive are linked to primary care at the point of testing.

\section{Materials and Methods}

The current investigation utilizes data from the University of Alabama at Birmingham 1917 Clinic HIV testing program (years 2007-2012) [35], which provides free POC testing using the OraQuick ADVANCE Rapid HIV-1/2 Antibody Test, which has $99.3 \%$ sensitivity and $99.8 \%$ specificity [22]. These tests were performed either within the context of a medical setting or at a community-based event. Testing services were initially offered by appointment and, with subsequent expanded staffing, on a walk-in basis. Based on the SAFE initiative constructs, POC testing was originally offered in this Deep South city as an ancillary service targeted toward sexual partners of HIV-infected patients attending the 1917 Clinic and was subsequently expanded to include nocost screening for any test-seeking community member [21]. Community-based locations for the 1917 Clinic-sponsored testing during the study period included university and other educational settings, health fairs, faith-based organizations, and rehabilitation centers. Outreach sites were commonly selected at the request of community leaders, based on evidence of need according to public health data, or in conjunction with a student or faith-based organization impacted by HIV, including African-American and LGBT student groups and congregations. Nested within a university based academic medical center with numerous healthcare personnel and a preexisting sexual health peer educator and internship program, the HIV testing and linkage team for this project expanded to include more than 150 trained staff and student volunteers.

OraQuick POC rapid HIV Tests were performed using a protocol based on the Health-Belief Model (HBM) by Hochbaum and Rosenstock $(1958,1960)$, a well-recognized conceptual framework for health-related interventions [36]. The HBM based risk assessment interview focused on perceived risk, benefits and barriers, enabling factors, cues to action, and self-efficacy and was created and merged with CDC and state of Alabama HIV serology templates to accommodate state reporting guidelines. Trained counselors used this standard form to describe HIV transmission, review Alabama reporting requirements for positive results, and collect pertinent data elements. Demographics, HIV and STI screening and risk history, and partner characteristics were included in each interview. Counselors used the 20-minute time period during which test results were processed for conveying tailored messages geared toward self-efficacy and discussion of risk reduction.

OraQuick Volunteer Testing Counselors were trained to provide confidential and compassionate HIV testing, counseling, and linkage services within the context of a nonjudgmental environment. The objectives were to promote self-awareness of risk-taking behavior, decrease risk of HIV infection by addressing identified barriers and benefits for modifying behavior, and provide linkage to care for persons 
TABLE 1: Test results of clients who presented for HIV testing at clinic and outreach settings by year, N (\%), 2007-2012.

\begin{tabular}{lccccccc}
\hline \multirow{2}{*}{ Result } & \multicolumn{3}{c}{ Test year } & \multicolumn{2}{c}{ Total } \\
& 2007 & 2008 & 2009 & 2010 & 2011 & $532(96)$ \\
Negative & $684(96)$ & $723(97)$ & $572(96)$ & $459(95)$ & $533(95)$ & $2603(96)$ \\
Positive & $25(4)$ & $26(3)$ & $26(4)$ & $23(5)$ & $26(5)$ & $22(4)$ & $148(4)$ \\
\hline Total & 709 & 749 & 598 & 482 & 559 & 554 & 3,651 \\
\hline
\end{tabular}

TABLE 2: Sociodemographic characteristics of clients who presented for HIV POC testing by test result, AL, 2007-2012.

\begin{tabular}{|c|c|c|c|c|}
\hline Characteristic & $\begin{array}{c}\text { Total } \\
N=3,651 \\
n(\%)\end{array}$ & $\begin{array}{l}\text { HIV positive } \\
\qquad \begin{array}{c}N=148 \\
n(\%)\end{array}\end{array}$ & $\begin{array}{l}\text { HIV negative } \\
\begin{array}{c}N=3,503 \\
n(\%)\end{array}\end{array}$ & $P$ value ${ }^{\mathrm{a}}$ \\
\hline Age, years & & & & $0.05^{*}$ \\
\hline 14 to 29 & $2,103(58)$ & $74(50)$ & $2,029(58)$ & \\
\hline$\geq 30$ & $1,535(42)$ & $74(50)$ & $1,461(42)$ & \\
\hline Sex & & & & $<0.001^{*}$ \\
\hline Male & $1,756(48)$ & $116(78)$ & $1,640(47)$ & \\
\hline Female & $1,887(52)$ & $32(22)$ & $1,855(53)$ & \\
\hline Race & & & & $0.03^{*}$ \\
\hline Black/AA & $2,126(59)$ & $100(68)$ & $2026(59)$ & \\
\hline White & $1,287(36)$ & $45(31)$ & $1242(36)$ & \\
\hline Other & $171(5)$ & $2(1)$ & $169(5)$ & \\
\hline Race/gender & & & & $<0.001^{*}$ \\
\hline White female & $535(15)$ & $4(3)$ & $531(15)$ & \\
\hline White male & $750(21)$ & $41(28)$ & $709(21)$ & \\
\hline Non-white female & $1,318(37)$ & $28(19)$ & $1290(38)$ & \\
\hline Non-White male & $978(27)$ & $74(50)$ & $904(26)$ & \\
\hline Ethnicity & & & & 0.80 \\
\hline Hispanic & $101(3)$ & $3(2)$ & $98(3)$ & \\
\hline Non-Hispanic & $3,188(97)$ & $140(98)$ & $3,048(97)$ & \\
\hline Residence & & & & 0.84 \\
\hline Alabama & $3,466(96)$ & $141(96)$ & $3,325(96)$ & \\
\hline Out of state & $160(4)$ & $6(4)$ & $154(4)$ & \\
\hline
\end{tabular}

${ }^{*}$ Statistically significant at 0.05 level.

AA: African American; HIV: Human Immunodeficiency Virus; POC: point of care.

Note: missing/unknown data (not shown) has been excluded for calculating percentages and $P$ value.

${ }^{a} P$ value (two tailed): Pearson chi-square test or Fisher's exact test.

testing HIV positive. For this investigation, linkage was defined as confirmed attendance at one or more HIV primary care visits during the 12-month period after receiving positive results.

The colocation of POC testing and linkage services within a Ryan White HIV clinic may have helped to alleviate barriers to care for those who received a preliminary positive result with OraQuick Rapid technology. While not all clients qualified for care at the 1917 Clinic due to insurance limitations, service area restrictions, or out-of-state residence, the goal of the POC testing team was to facilitate a primary care linkage visit within 90 days of a positive test. Consistent with the NHAS to link positive persons to medical care at time of diagnosis [25], the team made every attempt to establish a first intake appointment as part of same-day posttest counseling. Clients who decided to enroll in care at the 1917 Clinic were immediately scheduled for a linkage visit with a Project CONNECT (Client-Oriented New Patient
Navigation to Encourage Connection to Treatment) staff member or case manager [37, 38]. In the alternative, clients who tested positive who elected to receive care elsewhere were followed by testing staff and their county health department until they obtained a confirmatory test and first medical appointment at another HIV clinic or by private physician. All test seekers were provided with direct access to a member of the 1917 Clinic Prevention Education staff as needed during the linkage process. For those who encountered barriers to linking to care, the 1917 Clinic testing team worked in partnership with the local health department, state peer mentors, and in-house staff to facilitate linkage and maintain contact over a 12-month period.

Our team evaluated a database of 4,397 interview responses from persons at least of 14 years of age who presented for a rapid POC HIV test over a six-year period, from January 1, 2007, to December 31, 2012. Of these, 448 clients had more than one HIV test during the study period; 


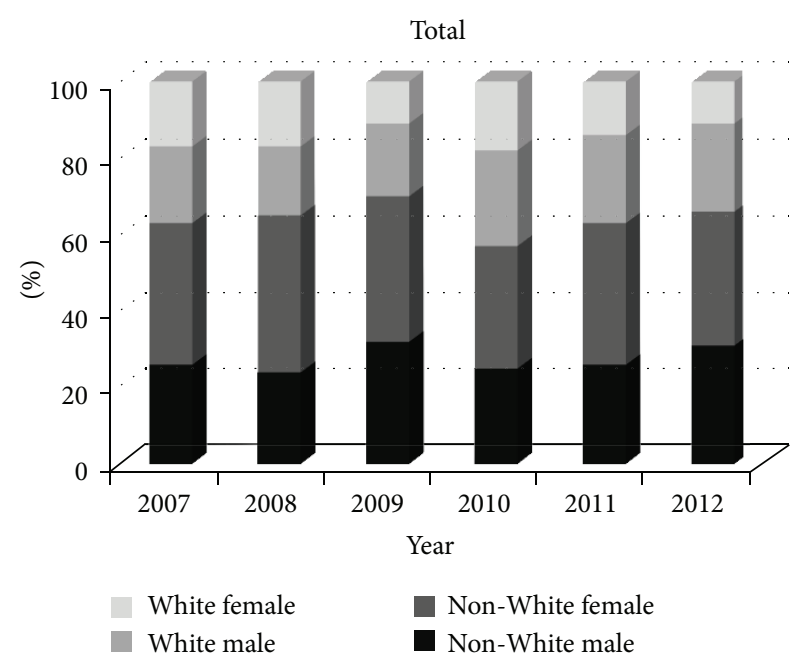

(a)

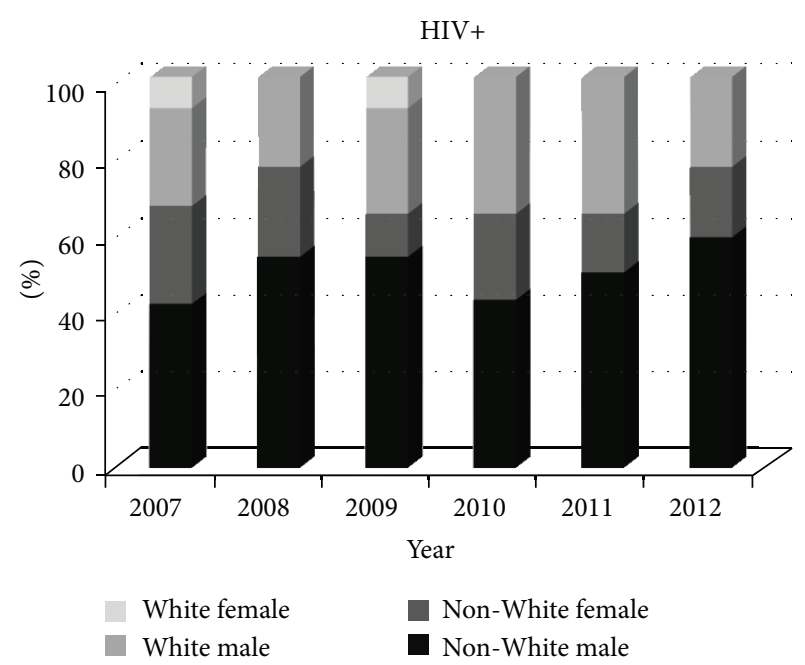

(b)

Figure 1: Race-Gender Distribution by year, AL, 2007-2012.

only the initial test was used in this analysis, yielding 3,651 unique client interviews. As clients were able to refuse any question, results are reported using a denominator of persons who were willing to provide answers to interviewers for that item.

The sample had a total of 148 (4\%) positive results; three of these were regarded as initial false negatives (though negative at first contact interview, a subsequent test was positive within 90 days of the first test result) and were excluded for subsequent analysis. Thus, linkage to care data were included for 145 individuals who had at least ninety days of follow up since a positive test result. The study protocol for this investigation was approved by the UAB Institutional Review Board.

2.1. Statistical Analysis. In addition to reporting overall demographics of the population, clients were grouped by HIV test result into positive or negative for descriptive reporting; sample size was 3,651. Continuous variables were described as median with range due to skewed distribution. Categorical variables were reported using frequencies with percentages and compared using Pearson's chi-square test or Fisher's exact test. Statistical significance was set at 0.05 (two-tailed test). Data were analyzed using SAS statistical software (version 9.3; Cary, NC) and Microsoft Excel (for figures).

\section{Results and Discussion}

3.1. Results. Table 1 shows the POC test results by year for the 3,651 included client interviews, with a positivity rate of $3-5 \%$ each, and $4 \%$ of the total sample testing positive. Demographic characteristics of the overall population are shown in Table 2. Median client age was 27 years (range 14 to 79 years), with $58 \%$ under 30 years of age; median age of HIV positive clients was 29 years, while that of HIV negative clients was 27 years $(P=0.003)$. Gender distribution was nearly equal. The majority was Black/African American (Black/AA). Nearly all clients were residents of Alabama.

A race/gender variable was created so that interview responses for those who reported a race other than Black/AA or White were not excluded from the analysis. As the literature has described an increase in new infections among Black/AA and minority males domestically, the race/gender variable was grouped (White and non-White) to indicate whether local sample was reflective of this trend [39-43]. As illustrated in Figure 1, non-White males accounted for approximately one-quarter to one-third of the testing population each year, but were overrepresented in the positive population across years. While non-White males represented half of the positive results, non-White females accounted for less than one-fifth of the positive results. White males represented an additional $28 \%$ of the positive sample. Reported characteristics of the population were grouped according to test result. Forty-seven percent $(N=1,723)$ of the overall population was tested at an outreach event; case finding at outreach events was less than $1 \%$. While approximately twothirds of the negative sample reported a previous HIV test at another location, $89 \%$ of those who tested positive reported a previous negative result, with $41 \%$ of positive clients reporting an HIV test in the previous six months (not shown).

While $16 \%$ of all clients reported having ever been tested and diagnosed with another sexually transmitted infection (STI), nearly one-third of those who tested positive reported a history of STI (Table 3). Men who have sex with men (MSM) represented $58 \%$ of the positive population. For clients who discussed condom utilization, significant $(P=$ 0.001 ) differences were observed, as $25 \%$ of negative and $15 \%$ of persons who tested positive reported consistent condom use (Table 3). For persons who reported any condom use, $44 \%$ of negative and $60 \%$ of positive clients reported condom utilization less than $100 \%$ of the time during vaginal or anal intercourse.

Among persons who responded to items about partner characteristics, $27 \%$ of persons who tested positive versus 
TABLE 3: Reported characteristics of clients who presented for HIV POC testing by test result, AL, 2007-2012.

\begin{tabular}{|c|c|c|c|c|}
\hline Characteristic & $\begin{array}{c}\text { Total } \\
N=3,651 \\
n(\%)\end{array}$ & $\begin{array}{c}\text { HIV positive } \\
\qquad \begin{array}{c}N=148 \\
n(\%)\end{array}\end{array}$ & $\begin{array}{l}\text { HIV negative } \\
\begin{array}{c}N=3,503 \\
n(\%)\end{array}\end{array}$ & $P$ value $^{\mathrm{a}}$ \\
\hline \multicolumn{5}{|l|}{ HIV testing } \\
\hline Test location & & & & $<0.001^{*}$ \\
\hline Outreach & $1,723(47)$ & $8(5)$ & $1,715(49)$ & \\
\hline HIV clinic & $1,896(52)$ & $140(95)$ & $1,756(51)$ & \\
\hline Previously tested & & & & $<0.001^{*}$ \\
\hline No & $1,241(34)$ & $16(11)$ & $1,225(35)$ & \\
\hline Yes & $2,378(66)$ & $131(89)$ & $2,247(65)$ & \\
\hline \multicolumn{5}{|l|}{ Participant risk factors } \\
\hline History of STI ${ }^{\mathrm{b}}$ & & & & $<0.001^{*}$ \\
\hline Yes & $491(16)$ & $36(30)$ & $455(16)$ & \\
\hline No & $2,482(83)$ & $84(70)$ & $2,398(84)$ & \\
\hline Unsure & $14(1)$ & $0(0)$ & $14(<1)$ & \\
\hline $\mathrm{MSM}^{\mathrm{b}}$ & & & & $<0.001^{*}$ \\
\hline Yes & $550(19)$ & $70(58)$ & $480(17)$ & \\
\hline No & $2,346(80)$ & $49(41)$ & $2,297(82)$ & \\
\hline Unsure & $30(1)$ & $1(1)$ & $29(1)$ & \\
\hline History of illicit drug use $\mathrm{e}^{\mathrm{c}}$ & & & & 0.26 \\
\hline Yes & $642(21)$ & $31(26)$ & $611(21)$ & \\
\hline No & $2,345(79)$ & $90(74)$ & $2,255(79)$ & \\
\hline Alcohol use & & & & 0.51 \\
\hline Yes & $2,100(70)$ & $89(73)$ & $2,011(70)$ & \\
\hline No & $889(30)$ & $33(27)$ & $856(30)$ & \\
\hline Condom utilization & & & & $0.001^{*}$ \\
\hline No condom use & $889(31)$ & $29(25)$ & $860(31)$ & \\
\hline Condom use $<100 \%$ & $1,276(44)$ & $71(60)$ & $1,205(44)$ & \\
\hline Condom use $=100 \%$ & $719(25)$ & $18(15)$ & $701(25)$ & \\
\hline
\end{tabular}

${ }^{*}$ Statistically significant at 0.05 level.

HIV: Human Immunodeficiency Virus; MSM: men having sex with men; POC: point of care; STI: sexually transmitted infections. Note: missing/unknown data (not shown) has been excluded for calculating percentages and $P$ value.

${ }^{a} P$ value (two tailed): Pearson chi-square test or Fisher's exact test.

b"No" and "unsure" categories combined to calculate $P$ value.

${ }^{c}$ Excluding marijuana.

$38 \%$ who tested negative reported none or a single partner (Table 4). HIV positive persons were significantly more likely to have 10 or more partners than HIV negative persons $(P=0.01)$. Partner status was also significant, as $40 \%$ of the positive clients had a known HIV positive partner as compared to $13 \%$ of the negative clients $(<0.001)$; overall $16 \%$ were unsure of their partner's HIV status (HIV positive $=21 \%$ versus HIV negative $=16 \%$ ) (Table 4 ).

Fourteen percent of clients who tested positive were motivated to be tested by a self-reported high risk event or behavior (Table 4). Forty-two percent of those who were positive listed their motivation as planned or routine serostatus update. One-third of those who tested positive were motivated by a specific referral for HIV testing as compared to $10 \%$ in HIV negative clients $(P<0.001)$.

Of the 145 persons who tested positive at POC and were eligible for linkage follow-up, 124 (86\%) had confirmed linkages to care, while the remaining 21 could not be confirmed as having attended a primary care visit in the 12 months following the positive result (unknown/unconfirmed). Of the 145 HIV positive persons, 115 elected to receive care at the 1917 Clinic and were immediately referred to Project CONNECT intake staff for a linkage visit. Time from positive test date to the arrived CONNECT intake visit was a median of five days (range $=0-378$ days). Of the 115 new clients who completed a CONNECT intake, $97 \%(n=112)$ arrived for a primary care visit at the 1917 Clinic. Median time from positive test to first arrived primary care appointment was 41 days, with $83 \%$ arriving for a first primary care visit within 90 days and an additional 13\% arriving within 180 days of first positive test (Figure 2). In addition to these 112 clients, 12 clients confirmed that they had accessed HIV primary care at another medical facility. Thus, 124 (86\%) of the 145 HIV positive results were classified as confirmed linkage to care. Of the 124 clients linked to care, most were residents of Alabama (Table 5).

Significant differences were found between the linked to care and "unknown/unconfirmed" linkage groups with 
TABLE 4: Reported participant and partner characteristics of clients who presented for HIV POC testing by test result, AL, $2007-2012$.

\begin{tabular}{|c|c|c|c|c|}
\hline Characteristic & $\begin{array}{c}\text { Total } \\
N=3,651 \\
n(\%)\end{array}$ & $\begin{array}{c}\text { HIV positive } \\
N=148 \\
n(\%)\end{array}$ & $\begin{array}{c}\text { HIV negative } \\
\begin{array}{c}N=3,503 \\
n(\%)\end{array}\end{array}$ & $P$ value \\
\hline Number of sexual partners & & & & $0.01^{*}$ \\
\hline 0 to 1 & $1,099(37)$ & $33(27)$ & $1,066(38)$ & \\
\hline 2 to 9 & $1,563(53)$ & $67(56)$ & $1,496(53)$ & \\
\hline 10 or more & $286(10)$ & $20(17)$ & $266(9)$ & \\
\hline Partner HIV positive ${ }^{\mathrm{b}}$ & & & & $<0.001^{*}$ \\
\hline Yes & $477(14)$ & $57(40)$ & $420(13)$ & \\
\hline No & $2,379(70)$ & $56(39)$ & $2,323(71)$ & \\
\hline Unsure & $561(16)$ & $30(21)$ & $531(16)$ & \\
\hline Partner is a clinic patient ${ }^{\mathrm{b}}$ & & & & $<0.001^{*}$ \\
\hline Yes & $348(12)$ & $33(26)$ & $315(11)$ & \\
\hline No & $2,495(82)$ & $83(66)$ & $2,412(83)$ & \\
\hline Unsure & $186(6)$ & $10(8)$ & $176(6)$ & \\
\hline Partner is $\mathrm{MSM}^{\mathrm{b}}$ & & & & $<0.001^{*}$ \\
\hline Yes & $566(21)$ & $68(58)$ & $498(19)$ & \\
\hline No & $1,936(71)$ & $41(35)$ & $1,895(73)$ & \\
\hline Unsure & $219(8)$ & $8(7)$ & $211(8)$ & \\
\hline \multicolumn{5}{|l|}{ Motivation for testing } \\
\hline High risk event & $358(13)$ & $16(14)$ & $342(13)$ & 0.76 \\
\hline Status/health update & $1,412(50)$ & $50(42)$ & $1,362(50)$ & 0.10 \\
\hline Convenience/ease & $527(19)$ & $5(4)$ & $522(19)$ & $<0.001^{*}$ \\
\hline Requirement for work/travel & $118(4)$ & $4(3)$ & $114(4)$ & 1.00 \\
\hline Referred for testing & $318(11)$ & $39(33)$ & $279(10)$ & $<0.001^{*}$ \\
\hline Other & $99(4)$ & $4(3)$ & $95(4)$ & 1.00 \\
\hline \multicolumn{5}{|l|}{ Referral source } \\
\hline Health professional & $205(7)$ & $29(25)$ & $176(7)$ & $<0.001^{*}$ \\
\hline Public campaign/media & $1,338(48)$ & $17(15)$ & $1,321(49)$ & $<0.001^{*}$ \\
\hline Self & $238(8)$ & $5(4)$ & $233(9)$ & 0.10 \\
\hline Partner & $267(9)$ & $20(17)$ & $247(9)$ & $0.004^{*}$ \\
\hline Friend/family member & $617(22)$ & $38(32)$ & $579(21)$ & $0.005^{*}$ \\
\hline Other/nonspecific & $153(5)$ & $8(7)$ & $145(5)$ & 0.49 \\
\hline
\end{tabular}

${ }^{*}$ Statistically significant at 0.05 level.

Note: missing/unknown data (not shown) has been excluded for calculating percentages and $P$ value.

HIV: Human Immunodeficiency Virus; MSM: men having sex with men; POC: point of care.

${ }^{a} P$ value (two tailed): Pearson chi-square test or Fisher's exact test.

b"Unsure" category excluded for calculating $P$ value.

regard to gender and race-gender (Table 5). Being tested at an outreach event was significantly associated with "unknown/ unconfirmed" linkage to care $(P=0.01)$ (Table 6). We did not find significant differences between the linked to care and "unknown/unconfirmed" groups with regard to previous HIV testing, condom utilization, or risk factors such as history of STI, MSM, illicit drug, or alcohol use (Table 6). Similarly, no significant differences were observed with regard to number of sexual partners or other partnerrelated characteristics (Table 7 ). Also, motivation for testing and referral source did not differ significantly between the two groups, with the exception that $38 \%$ of the linked clients were motivated by a specific referral for testing as compared to $11 \%$ in the "unknown/unconfirmed" group $(P=0.03)$ (Table 7).

3.2. Discussion. Overall, more females than males were present in the testing sample (52\% versus $48 \%$ ), and higher numbers of Black/AA participants than White (59\% versus $36 \%$ ), which is representative of the surrounding Birmingham, AL, metropolitan area and local census figures [44]. Overall trends showed a positivity rate of 3-5\% across all years and high rates of reported history of HIV testing across groups.

Non-White males accounted for one-quarter of overall test-seeking clients and bore the majority of positive results, 
TABLE 5: Sociodemographic characteristics of clients who presented for HIV POC testing by linkage, AL, 2007-2012.

\begin{tabular}{|c|c|c|c|c|}
\hline \multirow[b]{2}{*}{ Characteristic } & \multicolumn{4}{|c|}{ Linked to care } \\
\hline & $\begin{array}{c}\text { Total } \\
N=145 \\
n(\%)\end{array}$ & $\begin{array}{c}\text { Confirmed } \\
N=124 \\
n(\%)\end{array}$ & $\begin{array}{l}\text { Unknown/unconfirmed } \\
\qquad \begin{array}{c}N=21 \\
n(\%)\end{array}\end{array}$ & $P$ value $^{\mathrm{a}}$ \\
\hline Age, years & & & & 0.54 \\
\hline 14 to 29 & $74(51)$ & $62(50)$ & $12(57)$ & \\
\hline$\geq 30$ & $71(49)$ & $62(50)$ & $9(43)$ & \\
\hline Sex & & & & $0.02^{*}$ \\
\hline Male & $113(78)$ & $101(81)$ & $12(57)$ & \\
\hline Female & $32(22)$ & $23(18)$ & $9(43)$ & \\
\hline Race $^{b}$ & & & & 0.07 \\
\hline Black/AA & $99(69)$ & $81(66)$ & $18(86)$ & \\
\hline White & $43(30)$ & $40(32)$ & $3(14)$ & \\
\hline Other & $2(1)$ & $2(2)$ & $0(0)$ & \\
\hline Race/gender & & & & $0.05^{*}$ \\
\hline White female & $4(3)$ & $4(3)$ & $0(0)$ & \\
\hline White male & $39(27)$ & $36(29)$ & $3(14)$ & \\
\hline Non-white female & $28(19)$ & $19(16)$ & $9(43)$ & \\
\hline Non-white male & $73(51)$ & $64(52)$ & $9(43)$ & \\
\hline Ethnicity & & & & 1.00 \\
\hline Hispanic & $3(2)$ & $3(3)$ & $0(0)$ & \\
\hline Non-Hispanic & $137(98)$ & $116(97)$ & $21(100)$ & \\
\hline Residence & & & & 1.00 \\
\hline Alabama & $138(96)$ & $119(96)$ & $19(95)$ & \\
\hline Out of state & $6(4)$ & $5(4)$ & $1(5)$ & \\
\hline
\end{tabular}

${ }^{*}$ Statistically significant at 0.05 level.

AA: African American; HIV: Human Immunodeficiency Virus.

Note: Missing/unknown data (not shown) has been excluded for calculating percentages and $P$ value.

${ }^{a} P$ value (two tailed): Pearson chi-square test or Fisher's exact test.

${ }^{b}$ Categories "White" and "other" combined to calculate $P$ value.

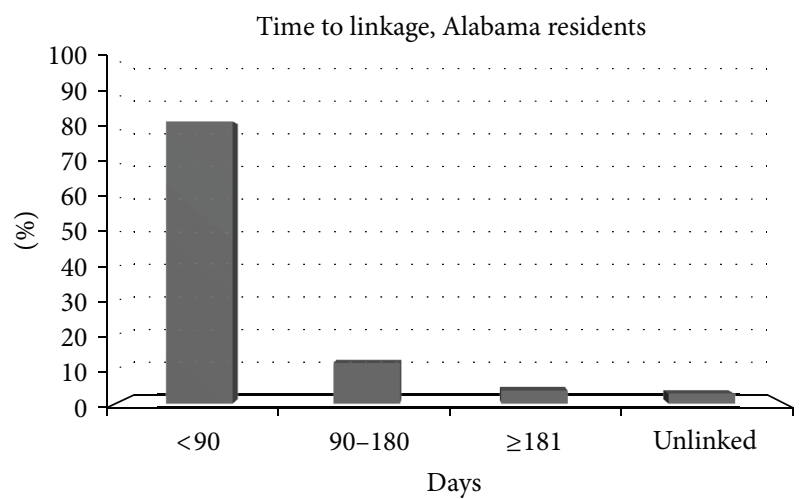

FIGURE 2: Time to Linkage in days for HIV+ Alabama Residents who participated in an integrated linkage program, AL, 2007-2012.

as shown in Figure 1, and more than half of persons who tested positive were MSM. This is consistent with the current HIV literature that reflects an overrepresentation of positive MSM and minority males, as well as disparities, discrimination, and social inequalities that may contribute to disproportionately high rates of HIV/AIDS in the Black/AA population in the South $[40,45,46]$. Additionally, as only $14 \%$ of persons who tested positive in this sample were motivated for testing by perceived high risk behavior, and timely utilization of HIV testing services among MSM has been observed in other studies, it may be valuable to expand high impact prevention strategies for this population by providing individual and couples testing services for MSM as well as offering PrEP to persons with higher risk behaviors [47-49].

Self-reported motivation and referral source for testing were of particular interest and, as with previous investigations of this population, convenience and individual "routine" status updates were chief motivators for testing in this population overall [35]. This highlights the importance of access to screening personnel across POC venues, as $4 \%$ of persons who tested positive and $19 \%$ of those who were negative reported that they were motivated by the convenience of time or location, and half of those who tested negative, along with $42 \%$ of positive persons, reported that they were tested as part of routine HIV status checks. Though anticipated, it is also noteworthy that referrals were a foremost testing 
TABLE 6: Reported characteristics of clients who presented for HIV POC testing by linkage, AL, 2007-2012.

\begin{tabular}{|c|c|c|c|c|}
\hline \multirow[b]{2}{*}{ Characteristic } & \multicolumn{4}{|c|}{ Linked to care } \\
\hline & $\begin{array}{c}\text { Total } \\
N=145 \\
n(\%)\end{array}$ & $\begin{array}{c}\text { Confirmed } \\
\begin{array}{c}N=124 \\
n(\%)\end{array}\end{array}$ & $\begin{array}{l}\text { Unknown/unconfirmed } \\
\qquad \begin{array}{c}N=21 \\
n(\%)\end{array}\end{array}$ & $P$ value $^{\mathrm{a}}$ \\
\hline \multicolumn{5}{|l|}{ HIV testing } \\
\hline Test location & & & & $0.01^{*}$ \\
\hline Outreach & $7(5)$ & $3(2)$ & $4(19)$ & \\
\hline HIV clinic & $138(95)$ & $121(98)$ & $17(81)$ & \\
\hline Previously tested & & & & 0.47 \\
\hline No & $16(11)$ & $15(12)$ & $1(5)$ & \\
\hline Yes & $128(89)$ & $108(88)$ & $20(95)$ & \\
\hline \multicolumn{5}{|l|}{ Participant risk factors } \\
\hline History of STI & & & & 0.39 \\
\hline Yes & $36(30)$ & $29(29)$ & $7(39)$ & \\
\hline No & $83(70)$ & $72(71)$ & $11(61)$ & \\
\hline $\mathrm{MSM}^{\mathrm{b}}$ & & & & 0.32 \\
\hline Yes & $68(58)$ & $59(60)$ & $9(47)$ & \\
\hline No & $49(41)$ & $39(39)$ & $10(53)$ & \\
\hline Unsure & $1(1)$ & $1(1)$ & $0(0)$ & \\
\hline History of illicit drug use $e^{c}$ & & & & 1.00 \\
\hline Yes & $31(26)$ & $26(26)$ & $5(26)$ & \\
\hline No & $88(74)$ & $74(74)$ & $14(74)$ & \\
\hline Alcohol use & & & & 0.60 \\
\hline Yes & $88(73)$ & $75(74)$ & $13(68)$ & \\
\hline No & $32(27)$ & $26(26)$ & $6(32)$ & \\
\hline Condom utilization & & & & 0.51 \\
\hline No condom use & $28(24)$ & $26(26)$ & $2(13)$ & \\
\hline Condom use $<100 \%$ & $71(61)$ & $59(59)$ & $12(75)$ & \\
\hline Condom use $=100 \%$ & $17(15)$ & $15(15)$ & $2(13)$ & \\
\hline
\end{tabular}

${ }^{*}$ Statistically significant at 0.05 level.

HIV: Human Immunodeficiency Virus; MSM: men having sex with men; POC: point of care; STI: sexually transmitted infections.

Note: missing/unknown data (not shown) has been excluded for calculating percentages and $P$ value.

${ }^{a} P$ value (two tailed): Pearson chi-square test or Fisher's exact test.

b"No" and "unsure" categories combined to calculate $P$ value.

${ }^{c}$ Excluding marijuana.

motivator for persons who were positive, as one-third of those who received positive results reported that they had received a specific referral for HIV screening. Referrals from a healthcare facility (25\%), friend, or family member (32\%) accounted for more than half (57\%) of all positive results, which may reflect promise for targeted campaigns aimed toward normalizing screening. Improving HIV status awareness may be particularly crucial for reducing risk in this population, as $21 \%$ of the HIV positive persons in this sample were unsure of their partner HIV status, more than half reported inconsistent condom use, and nearly a quarter of positive persons reported no condom use at all.

Primary limitations for this study included low representation of intravenous drug users and racial subgroups other than White and Black/African American. As an additional limitation was a reliance on the self-reported information that was gleaned via face-to-face testing and counseling interviews, low reporting of high risk drug behavior is not surprising. It is also important to note that, while $47 \%$ of individuals in the sample were tested through outreach, this yielded fewer positive results and confirmed linkages overall; a formal evaluation of outreach locations and processes will help to shape future efforts to respond to community requests for testing versus increasing targeted testing by area of demonstrated need. Outreach screening at regional health events may also have contributed to linkage challenges, as persons outside of the clinic service area may not have been eligible to link to care locally. Follow-up analyses for clients who did not attend a confirmed primary care visit and persons who were lost to follow up will provide supplementary insight on individual barriers to linkage. In addition, though this investigation utilized a convenience sample of 
TABLE 7: Reported participant and partner characteristics of clients who presented for HIV POC testing by linkage, AL, $2007-2012$.

\begin{tabular}{|c|c|c|c|c|}
\hline \multirow[b]{2}{*}{ Characteristic } & \multicolumn{4}{|c|}{ Linked to care } \\
\hline & $\begin{array}{c}\text { Total } \\
N=145 \\
n(\%)\end{array}$ & $\begin{array}{c}\text { Confirmed } \\
N=124 \\
n(\%)\end{array}$ & $\begin{array}{l}\text { Unknown/unconfirmed } \\
\qquad \begin{array}{c}N=21 \\
n(\%)\end{array}\end{array}$ & $P$ value $^{\mathrm{a}}$ \\
\hline Number of sexual partners & & & & 0.34 \\
\hline 0 to 1 & $33(28)$ & $28(28)$ & $5(28)$ & \\
\hline 2 to 9 & $66(55)$ & $58(57)$ & $8(44)$ & \\
\hline 10 or more & $20(17)$ & $15(15)$ & $5(28)$ & \\
\hline Partner HIV positive ${ }^{\mathrm{b}}$ & & & & 0.17 \\
\hline Yes & $56(40)$ & $51(43)$ & $5(25)$ & \\
\hline No & $56(40)$ & $46(38)$ & $10(50)$ & \\
\hline Unsure & $28(20)$ & $23(19)$ & $5(25)$ & \\
\hline Partner is a clinic patient $\mathrm{t}^{\mathrm{b}}$ & & & & 0.22 \\
\hline Yes & $33(27)$ & $30(28)$ & $3(16)$ & \\
\hline No & $82(66)$ & $67(64)$ & $15(79)$ & \\
\hline Unsure & $9(7)$ & $8(8)$ & $1(5)$ & \\
\hline Partner is $\mathrm{MSM}^{\mathrm{b}}$ & & & & 0.79 \\
\hline Yes & $66(57)$ & $56(58)$ & $10(53)$ & \\
\hline No & $41(36)$ & $34(36)$ & $7(37)$ & \\
\hline Unsure & $8(7)$ & $6(6)$ & $2(10)$ & \\
\hline \multicolumn{5}{|l|}{ Motivation for testing } \\
\hline High risk event & $16(14)$ & $14(14)$ & $2(11)$ & 1.00 \\
\hline Status/health update & $49(42)$ & $41(42)$ & $8(44)$ & 0.84 \\
\hline Convenience/ease & $5(4)$ & $2(2)$ & $3(17)$ & $0.03^{*}$ \\
\hline Requirement for work/travel & $4(3)$ & $3(3)$ & $1(6)$ & 0.50 \\
\hline Referred for testing & $39(34)$ & $37(38)$ & $2(11)$ & $0.03^{*}$ \\
\hline Other & $3(3)$ & $1(1)$ & $2(11)$ & 0.06 \\
\hline \multicolumn{5}{|l|}{ Referral source } \\
\hline Health professional & $28(24)$ & $24(25)$ & $4(22)$ & 1.00 \\
\hline Public campaign/media & $17(15)$ & $13(13)$ & $4(22)$ & 0.30 \\
\hline Self & $5(4)$ & $4(4)$ & $1(6)$ & 0.58 \\
\hline Partner & $19(17)$ & $17(18)$ & $2(11)$ & 0.73 \\
\hline Friend/family member & $38(33)$ & $32(33)$ & $6(33)$ & 0.98 \\
\hline Other/nonspecific & $8(7)$ & $7(7)$ & $1(6)$ & 1.00 \\
\hline
\end{tabular}

${ }^{*}$ Statistically significant at 0.05 level.

Note: missing/unknown data (not shown) has been excluded for calculating percentages and $P$ value.

HIV: Human Immunodeficiency Virus; MSM: men having sex with men; POC: point of care.

${ }^{a} P$ value (two tailed): Pearson chi-square test or Fisher's exact test.

b"Unsure" category excluded for calculating $P$ value.

test-seekers, this population was representative of the local urban community, and findings may be generalizable to other cities in the Southern Unites States.

The overall linkage rate for positive persons in this sample was $86 \%$, which included out-of-state residents, persons eligible for pediatric or obstetric care, persons who did not return for confirmatory results, individuals that were referred outside of Ryan White clinics, and persons who did not seek medical care locally, some of whom were lost to follow up despite staff contacts for up to 12 months after diagnosis. Significant differences in confirmed linkage were observed, specifically for non-White females, persons tested at outreach settings versus clinical settings, and for those who reported "convenience" as the motivation for testing. While we anticipated that some women may have elected to seek care with an existing gynecology or women's health provider/clinic, we did not predict that POC testing at outreach settings or test-seekers reporting being motivated by convenience would present significant unfavorable differences for confirmation of linkage in this cohort. Additional analyses on test-seeking clients who did not have a confirmed linkage to primary care are warranted, specifically to examine insurance status, clinical access, prior experience with the healthcare system, and novel mechanisms to minimize loss to follow up.

Challenges to linkage are not uncommon, and exploration of individual barriers to care, in combination with 
expanded integration of colocated linkage and surveillance efforts in the state or region, may improve linkage rates for marginalized, transient, and hard to reach individuals [31, 33, 50-52]. While further investigations may identify factors that keep some test-seekers from entering medical care following a positive diagnosis, these findings help to demonstrate the benefit of comprehensive POC testing and counseling and linkage services locally, as $97 \%$ of positive instate test seekers who participated in an immediate linkage program (median days $=5$ ) attended their first primary care visit (median days $=41$ ). For persons who were successfully linked locally, 96\% arrived for a first medical visit within 180 days of receiving a positive result, and $83 \%$ met the NHAS goal of linkage within 90 days [25], showing promise for integrated POC testing and linkage services.

\section{Conclusions}

Notable differences in race and gender, test location, and motivation for screening of persons who presented for POC HIV testing were observed for both test result and linkage to care in our investigation. Though rapid HIV, STI, and other POC testing innovations, including self- and in-home screenings, show promise for decreased diagnostic time with impressive results accuracy across locations [22, 31, 53-55], incorporating immediate active referrals and timely linkage to care services into these tactics is imperative for prompting early treatment initiation and improved outcomes [32, 5658]. In order to fully realize the potential of sophisticated screening and surveillance technologies, it is essential to examine the dynamic population of HIV POC test-seekers in the Unites States. Enhancing programming for integrated, comprehensive HIV testing and linkage services for populations outside of primary healthcare settings should continue as a principal approach toward meeting the goals of the National HIV/AIDS Strategy and improving serostatus awareness, linkage rates, and HIV outcomes.

\section{Disclosure}

This paper is derived from Dr. Zinski's original thesis project (ProQuest, 2008).

\section{Acknowledgments}

The authors wish to thank the entire 1917 Clinic Testing Team, whose dedication has expanded the reach of HIV education and awareness, POC testing and counseling, and linkage to HIV care in our community. Additionally, for their ongoing support of this endeavor, special thanks to Rev. Christopher M. Hamlin and Ms. Kelly L. Ross-Davis for championing the testing and linkage program with diligence and compassion since its inception in 2005.

\section{References}

[1] CDC, HIV Counseling and Testing at CDC-Supported SitesUnited States, 1999-2004, CDC, Atlanta, Ga, USA, 2006.
[2] CDC, Demonstration Projects for Health Departments and Community-Based Organizations (CBOs): Antiretroviral Treatment Access Study (ARTAS) II: Linkage to HIV Care, CDC, 2006.

[3] B. Branson, "Current HIV epidemiology and revised recommendations for HIV testing in health-care settings," Journal of Medical Virology, vol. 79, supplement 1, pp. S6-S10, 2007.

[4] A. Wurcel, T. Zaman, S. Zhen, and D. Stone, "Acceptance of HIV antibody testing among inpatients and outpatients at a public health hospital: a study of rapid versus standard testing," AIDS Patient Care and STDs, vol. 19, no. 8, pp. 499-505, 2005.

[5] "Vital signs: HIV infection, testing, and risk behaviors among youths-United States," Morbidity and Mortality Weekly Report, pp. 971-976, 2012.

[6] H. F. Raymond, T. Bingham, and W. McFarland, "Locating unrecognized HIV infections among men who have sex with men: San Francisco and Los Angeles," AIDS Education and Prevention, vol. 20, no. 5, pp. 408-419, 2008.

[7] R. P. Walensky, E. Losina, K. A. Steger-Craven, and K. A. Freedberg, "Identifying undiagnosed human immunodeficiency virus: the yield of routine, voluntary inpatient testing," Archives of Internal Medicine, vol. 162, no. 8, pp. 887-892, 2002.

[8] M. L. Campsmith, P. H. Rhodes, I. Hall, and T. A. Green, "Undiagnosed HIV prevalence among adults and adolescents in the United States at the end of 2006," Journal of Acquired Immune Deficiency Syndromes, vol. 53, no. 1, pp. 619-624, 2010.

[9] M. Chen, P. H. Rhodes, I. H. Hall et al., "Prevalence of undiagnosed HIV infection among persons aged $\geq 13$ yearsNational HIV Surveillance System, United States, 2005-2008," Morbidity and Mortality Weekly Report, vol. 61, supplement, pp. 57-64, 2012.

[10] L. Peralta, B. G. Deeds, S. Hipszer, and K. Ghalib, "Barriers and facilitators to adolescent HIV testing," AIDS Patient Care and STDs, vol. 21, no. 6, pp. 400-408, 2007.

[11] S. A. Rizza, R. J. MacGowan, D. W. Purcell, B. M. Branson, and Z. Temesgen, "HIV screening in the health care setting: status, barriers, and potential solutions," Mayo Clinic Proceedings, vol. 87, pp. 915-924, 2012.

[12] S. Schwarcz, T. A. Richards, H. Frank et al., "Identifying barriers to HIV testing: personal and contextual factors associated with late HIV testing," AIDS Care, vol. 23, no. 7, pp. 892-900, 2011.

[13] N. P. Pai, C. Vadnais, C. Denkinger, N. Engel, and M. Pai, "Pointof-care testing for infectious diseases: diversity, complexity, and barriers in low- and middle-income countries," PLOS Medicine, vol. 9, Article ID e1001306, 2012.

[14] CDC, HIV Testing Trends in the United States, 2000-2011, CDC, Atlanta, Ga, USA, 2013.

[15] C. Hanssens, "Legal and ethical implications of opt-out HIV testing," Clinical Infectious Diseases, vol. 45, supplement 4, pp. S232-S239, 2007.

[16] R. Bayer and G. M. Oppenheimer, "Routine HIV testing, public health, and the USPSTF - an end to the debate," The New England Journal of Medicine, vol. 368, pp. 881-884, 2013.

[17] E. G. Martin and B. R. Schackman, "Updating the HIV-testing guidelines-a modest change with major consequences," The New England Journal of Medicine, vol. 368, pp. 884-886, 2013.

[18] A. P. Mahajan, L. Stemple, M. F. Shapiro, J. B. King, and W. E. Cunningham, "Consistency of state statutes with the centers for disease control and prevention HIV testing recommendations for health care settings," Annals of Internal Medicine, vol. 150, no. 4, pp. 263-269, 2009. 
[19] A. Qaseem, V. Snow, P. Shekelle, R. Hopkins Jr., and D. K. Owens, "Screening for HIV in health care settings: a guidance statement from the American College of Physicians and HIV Medicine Association," Annals of Internal Medicine, vol. 150, no. 2, pp. 125-131, 2009.

[20] L. A. Kennedy, F. M. Gordin, and V. L. Kan, "Assessing targeted screening and low rates of HIV testing," American Journal of Public Health, vol. 100, no. 9, pp. 1765-1768, 2010.

[21] R. S. Janssen, D. R. Holtgrave, R. O. Valdiserri, M. Shepherd, H. D. Gayle, and K. M. De Cock, "The serostatus approach to fighting the HIV epidemic: prevention strategies for infected individuals," American Journal of Public Health, vol. 91, no. 7, pp. 1019-1024, 2001.

[22] OraSure Technologies, OraQuick ADVANCE Rapid HIV-1/2 Antibody Test. OraSure Technologies, OraSure Technologies, Bethlehem, Pa, USA, 2009.

[23] CDC, Quality Assurance Guidelines for Testing Using Rapid HIV Antibody Tests Waived Under the Clinical Laboratory Improvement Amendments of 1988, CDC, 2007.

[24] J. D. O'Neal, M. R. Golden, B. M. Branson, and J. D. Stekler, "HIV nucleic acid amplification testing versus rapid testing: it is worth the wait. Testing preferences of men who have sex with men," Journal of Acquired Immune Deficiency Syndromes, vol. 60, pp. el17-e120, 2012.

[25] Office of National AIDS Policy, National HIV/AIDS Strategy, The White House, Washington, DC, USA, 2010.

[26] S. D. Pinkerton and C. L. Galletly, "Reducing HIV transmission risk by increasing serostatus disclosure: a mathematical modeling analysis," AIDS and Behavior, vol. 11, no. 5, pp. 698-705, 2007.

[27] A. K. Sullivan, H. Curtis, C. A. Sabin, and M. A. Johnson, "Newly diagnosed HIV infections: review in UK and Ireland," British Medical Journal, vol. 330, no. 7503, pp. 1301-1302, 2005.

[28] G. Marks, N. Crepaz, J. W. Senterfitt, and R. S. Janssen, "Metaanalysis of high-risk sexual behavior in persons aware and unaware they are infected with HIV in the United States: implications for HIV prevention programs," Journal of Acquired Immune Deficiency Syndromes, vol. 39, no. 4, pp. 446-453, 2005.

[29] L. S. Weinhardt, "Effects of a detailed sexual behavior interview on perceived risk of HIV infection: preliminary experimental analysis in a high risk sample," Journal of Behavioral Medicine, vol. 25, no. 2, pp. 195-203, 2002.

[30] R. P. Walensky, A. D. Paltiel, E. Losina et al., "Test and treat DC: forecasting the impact of a comprehensive HIV strategy in Washington DC," Clinical Infectious Diseases, vol. 51, pp. 392400, 2010

[31] R. P. Walensky and I. V. Bassett, "HIV self-testing and the missing linkage," PLoS Medicine, vol. 8, no. 10, Article ID e1001101, 2011.

[32] C. Gopalappa, P. G. Farnham, A. B. Hutchinson, and S. L. Sansom, "Cost effectiveness of the National HIV/AIDS Strategy goal of increasing linkage to care for HIV-infected persons," Journal of Acquired Immune Deficiency Syndromes, vol. 61, pp. 99-105, 2012.

[33] J. Bertolli, R. L. Shouse, L. Beer et al., "Using HIV surveillance data to monitor missed opportunities for linkage and engagement in HIV medical care," The Open AIDS Journal, vol. 6, pp. 131-141, 2012.

[34] W. E. Norton, "An exploratory study to examine intentions to adopt an evidence-based HIV linkage-to-care intervention among state health department AIDS directors in the United States," Implementation Science, vol. 7, article 27, 2012.
[35] A. Zinski, Who is in a hurry for HIV test results? An exploration of presentation for Oraquick rapid result HIV antibody testing in urban clinical and outreach settings in Alabama. Dissertation Abstracts International: Section B: The Sciences and Engineering, ProQuest International, 2008.

[36] K. Glanz, B. Rimer, and F. Lewis, Health Behavior and Health Education: Theory, Research, and Practice, Wiley \& Sons, San Francisco, 2002.

[37] R. Wylie, M. Marler, H. Lin et al., "Project CONNECT: overcoming barriers to establishing HIV care," in Proceedings of the 4th International Conference on HIV Treatment Adherence, Miami, Fla, USA, 2009,.

[38] D. S. Batey, S. T. Lawrence, N. C. Godwin et al., "Psychosocial symptom differences among new patient groups at an HIV medical clinic: project CONNECT," in Society for Social Work and Research 14th Annual Conference, San Francisco, Ca, USA, 2010.

[39] Q. An, J. Prejean, and H. I. Hall, "Racial disparity in U.S. Diagnoses of acquired immune deficiency syndrome, 20002009," American Journal of Preventive Medicine, vol. 43, pp. 461466, 2012.

[40] T. A. Farley, "Sexually transmitted diseases in the Southeastern United States: location, race, and social context," Sexually Transmitted Diseases, vol. 33, no. 7, pp. S58-S64, 2006.

[41] V. Fuqua, Y.-H. Chen, T. Packer et al., "Using social networks to reach Black MSM for HIV testing and linkage to care," AIDS and Behavior, vol. 16, no. 2, pp. 256-265, 2012.

[42] N. T. Harawa, M. Leng, J. Kim, and W. E. Cunningham, "Racial/ethnic and gender differences among older adults in nonmonogamous partnerships, time spent single, and human immunodeficiency virus testing," Sexually Transmitted Diseases, vol. 38, no. 12, pp. 1110-1117, 2011.

[43] W. A. Hill and C. McNeely, "HIV/AIDS disparity between African-American and caucasian men who have sex with men: intervention strategies for the black church," Journal of Religion and Health, vol. 52, no. 2, pp. 475-487, 2011.

[44] US Department of Commerce, "Jefferson County QuickFacts from the US Census Bureau," in State and County QuickFacts. Data Derived From Population Estimates, American Community Survey, Census of Population and Housing, State and County Housing Unit Estimates, County Business Patterns, Nonemployer Statistics, Economic Census, Survey of Business Owners, Building Permits, Consolidated Federal Funds Report, US Census Bureau, 2013.

[45] N. Harawa and A. Adimora, "Incarceration, African Americans and HIV: advancing a research agenda," Journal of the National Medical Association, vol. 100, no. 1, pp. 57-62, 2008.

[46] D. Stratford, Y. Mizuno, K. Williams, C. Courtenay-Quirk, and A. O'Leary, "Addressing poverty as risk for disease: recommendations from CDC's consultation on microenterprise as HIV prevention," Public Health Reports, vol. 123, no. 1, pp. 9-20, 2008.

[47] M. Fisher, "Late diagnosis of HIV infection: major consequences and missed opportunities," Current Opinion in Infectious Diseases, vol. 21, no. 1, pp. 1-3, 2008.

[48] E. Girardi, C. A. Sabin, and A. D. Monforte, "Late diagnosis of HIV infection: epidemiological features, consequences and strategies to encourage earlier testing," Journal of Acquired Immune Deficiency Syndromes, vol. 46, supplement 1, pp. S3-S8, 2007.

[49] S. Schwarcz, L. Hsu, J. W. Dilley, L. Loeb, K. Nelson, and S. Boyd, "Late diagnosis of HIV infection: trends, prevalence, and 
characteristics of persons whose HIV diagnosis occurred within 12 months of developing AIDS," Journal of Acquired Immune Deficiency Syndromes, vol. 43, no. 4, pp. 491-494, 2006.

[50] W. M. El-Sadr, T. R. Gamble, and M. S. Cohen, "Linkage from HIV testing to care: a positive test often leads nowhere," Sexually Transmitted Diseases, vol. 40, pp. 26-27, 2013.

[51] S. S. Johnston, T. Juday, D. Seekins et al., "Patterns and correlates of linkage to appropriate HIV care after HIV diagnosis in the US Medicaid population," Sexually Transmitted Diseases, vol. 40, pp. 18-25, 2013.

[52] N. M. Zetola, K. Bernstein, K. Ahrens et al., "Using surveillance data to monitor entry into care of newly diagnosed HIV-infected persons: San Francisco, 2006-2007," BMC Public Health, vol. 9, article 17, 2009.

[53] N. P. Pai and M. Pai, "Point-of-care diagnostics for HIV and tuberculosis: landscape, pipeline, and unmet needs," Discovery Medicine, vol. 13, pp. 35-45, 2012.

[54] N. P. Pai, B. Balram, S. Shivkumar et al., "Head-to-head comparison of accuracy of a rapid point-of-care HIV test with oral versus whole-blood specimens: a systematic review and meta-analysis," The Lancet Infectious Diseases, vol. 12, no. 5, pp. 373-380, 2012.

[55] M. Schito, T. F. Peter, S. Cavanaugh et al., "Opportunities and challenges for cost-efficient implementation of new point-ofcare diagnostics for HIV and tuberculosis," Journal of Infectious Diseases, vol. 205, supplement 2, pp. S169-S180, 2012.

[56] M. S. Cohen, Y. Q. Chen, M. McCauley et al., "Prevention of HIV-1 infection with early antiretroviral therapy," The New England Journal of Medicine, vol. 365, pp. 493-505, 2011.

[57] J. A. Fleishman, B. R. Yehia, R. D. Moore, and K. A. Gebo, “The economic burden of late entry into medical care for patients with HIV infection," Medical Care, vol. 48, no. 12, pp. 1071-1079, 2010.

[58] C. S. Krawczyk, E. Funkhouser, J. M. Kilby, and S. H. Vermund, "Delayed access to HIV diagnosis and care: special concerns for the Southern United States," AIDS Care, vol. 18, 1, pp. S35-S44, 2006. 


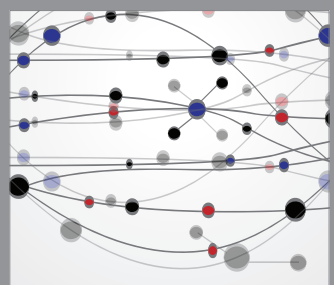

The Scientific World Journal
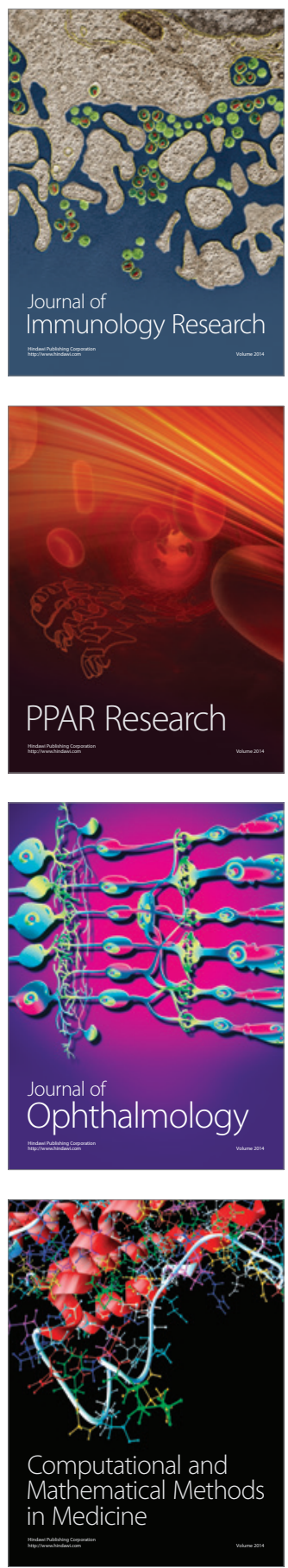

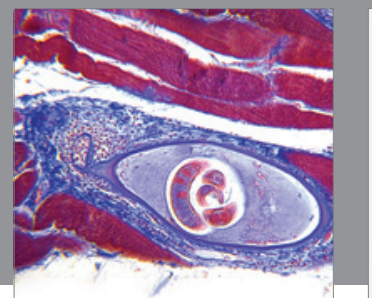

Gastroenterology

Research and Practice
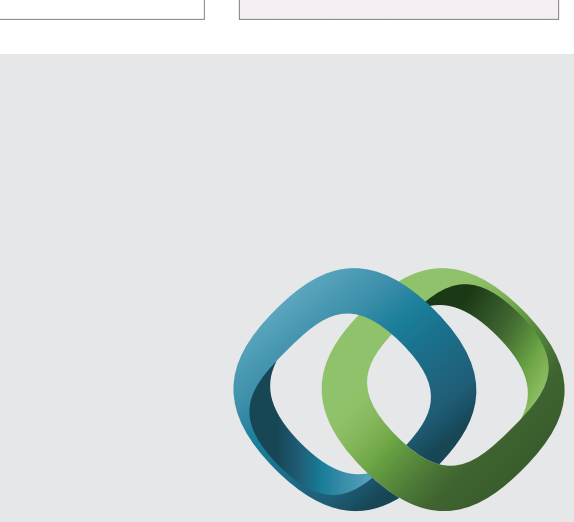

\section{Hindawi}

Submit your manuscripts at

http://www.hindawi.com
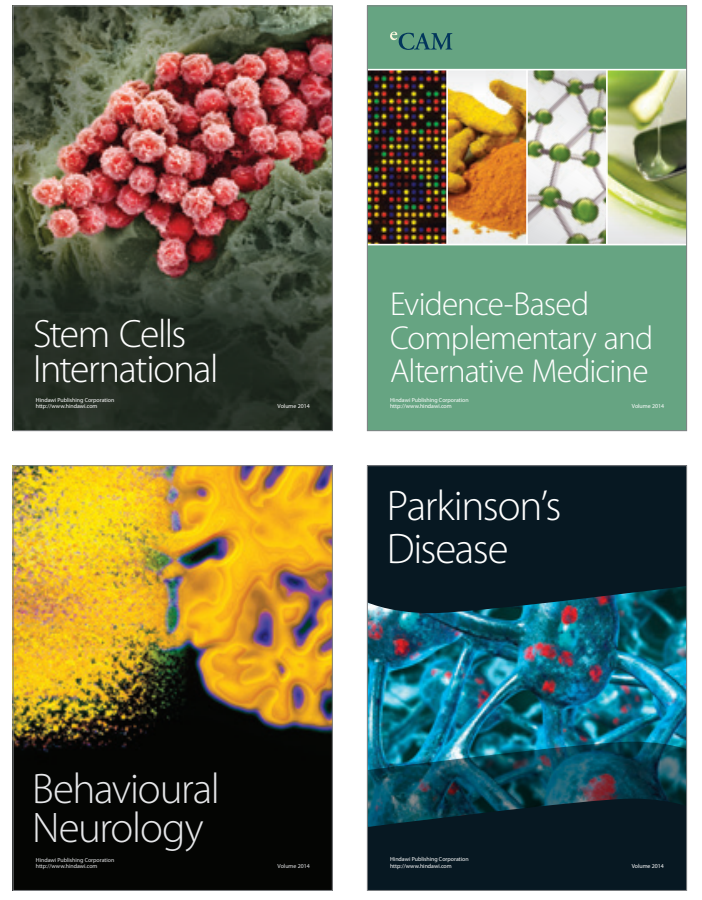
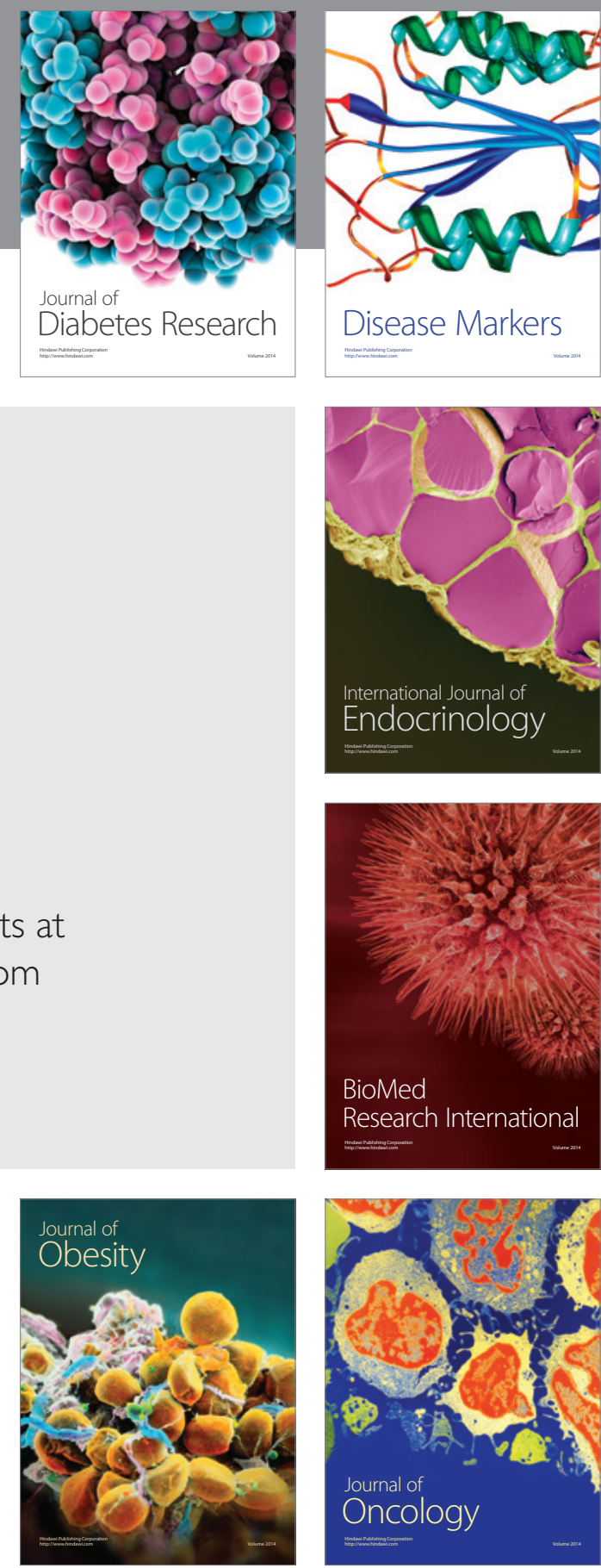

Disease Markers
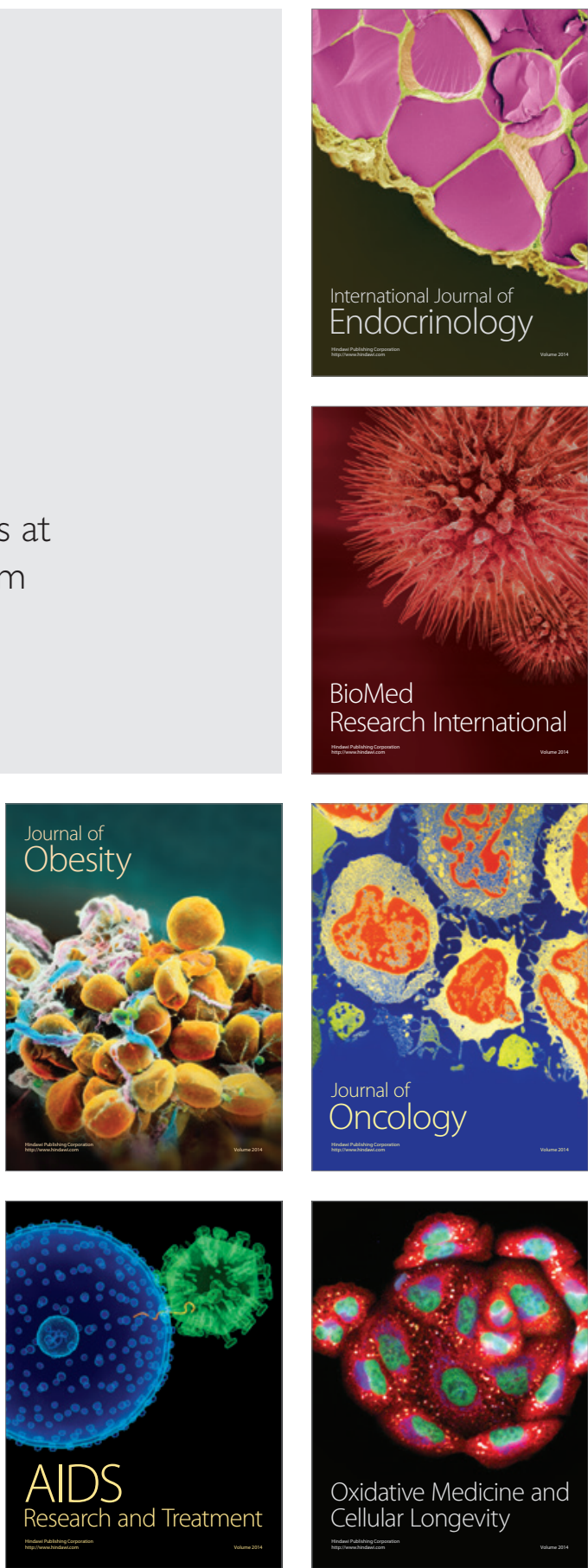